\title{
Possible $\Sigma^{0}-\Lambda$ mixing in the $Q C D$ sum rule
}

\author{
Shi-lin Zhu \\ Department of Physics, Peking University, Beijing, 100871, China \\ and Institute of Theoretical Physics, Academia Sinica, P.O. Box 2735, Beijing 100080, China
}

W-Y. P. Hwang

Department of Physics, National Taiwan University, Taipei, Taiwan 10764

Ze-sen Yang

Department of Physics, Peking University, Beijing, 100871, China

(Received 27 May 1997; published 8 January 1998)

\begin{abstract}
We calculate the on-shell $\Sigma^{0}-\Lambda$ mixing parameter $\theta$ with the method of the QCD sum rule. Our result is $\theta\left(m_{\Sigma 0}^{2}\right)=(-)(0.5 \pm 0.1) \mathrm{MeV}$. The electromagnetic interaction is not included. [S0556-2821(98)03103-8]

PACS number(s): 11.30.Hv, 11.55.Hx, 12.38.Lg, 14.20.Jn
\end{abstract}

Isospin independence and charge symmetry are only approximate in the strong interaction. It is believed that the up and down quark mass difference and the electromagnetic interaction cause all the isospin violations [1,2]. Experimentally a strong signature for $\rho^{0}-\omega$ mixing has been observed in the cross-section measurement of the reaction $e^{+} e^{-} \rightarrow \pi^{+} \pi^{-}$[3]. The effect from the electromagnetic interaction is of the opposite sign and much smaller than the experimentally determined $\left\langle\rho^{0}\left|H_{\mathrm{CSB}}\right| \omega\right\rangle$, while the current quark mass difference plays a dominant role [4]. Strong evidence for $\pi-\eta-\eta^{\prime}$ has also been obtained from studies of $\eta^{\prime}$ [5], $\psi^{\prime}$ [6], and $\psi$ [7] decays. In this work we study possible $\Sigma^{0}-\Lambda$ mixing with the method of QCD sum rules [8]. $\rho^{0}-\omega$ mixing has been analyzed within the same framework $[8,9]$. The isospin symmetry breaking sources in such an approach come from the current quark mass difference $\delta m=m_{u}-m_{d}$ $\neq 0$ and the quark condensate difference $\gamma$ $=\langle 0|\bar{d} d| 0\rangle /\langle 0|\bar{u} u| 0\rangle-1 \neq 0$. We do not take into account the electromagnetic interaction in the present work.

We may study $\Sigma^{0}-\Lambda$ mixing through the mixed propagator in the QCD vacuum

$$
\begin{aligned}
i \int d^{4} x e^{i p x}\left\langle 0\left|\Sigma^{0}(x) \bar{\Lambda}(0)\right| 0\right\rangle \\
=(-) \frac{\left(\hat{p}+m_{\Sigma^{0}}\right) \theta\left(\hat{p}+m_{\Lambda}\right)}{\left(p^{2}-m_{\Sigma^{0}}^{2}+i \epsilon\right)\left(p^{2}-m_{\Lambda}^{2}+i \epsilon\right)},
\end{aligned}
$$

where the mixing parameter $\theta\left(p^{2}\right)$ is defined through the following effective Lagragian:

$$
L^{\mathrm{mix}}=\theta\left(\bar{\Psi}_{\Sigma 0} \Psi_{\Lambda}+\bar{\Psi}_{\Lambda} \Psi_{\Sigma^{0}}\right)
$$

and may be measured using decays such as $\psi \rightarrow \Lambda \Sigma^{0}$ $+\bar{\Lambda} \Sigma^{0}$ in future experiments.

In order to calculate the mixing parameter we study the two-point correlator at the quark level as

$$
\Pi(p)=i \int d^{4} x\left\langle 0\left|T\left\{\eta_{\Sigma 0}(x), \bar{\eta}_{\Lambda}(0)\right\}\right| 0\right\rangle e^{i p \cdot x}
$$

$\eta_{\Sigma^{0}}$ and $\eta_{\Lambda}$ are the currents with $\Sigma^{0}$ and $\Lambda$ quantum numbers:

$$
\begin{aligned}
\eta_{\Sigma^{0}}(x)= & \epsilon^{a b c} \frac{1}{\sqrt{2}}\left\{\left[u^{a T}(x) C \gamma_{\mu} d^{b}(x)\right] \gamma_{5} \gamma^{\mu} s^{c}(x)\right. \\
& \left.+\left[d^{a T}(x) C \gamma_{\mu} u^{b}(x)\right] \gamma_{5} \gamma^{\mu} s^{c}(x)\right\}, \\
\eta_{\Lambda}(x)= & \epsilon^{a b c} \sqrt{\frac{2}{3}}\left\{\left[u^{a T}(x) C \gamma_{\mu} s^{b}(x)\right] \gamma_{5} \gamma^{\mu} d^{c}(x)\right. \\
& \left.-\left[d^{a T}(x) C \gamma_{\mu} s^{b}(x)\right] \gamma_{5} \gamma^{\mu} u^{c}(x)\right\},
\end{aligned}
$$

where $u^{a}(x), T$, and $C$ are the quark field, the transpose, and the charge conjugate operators. $a, b, c$ are the color indices. The interpolating currents couple to the baryon states with the overlap amplititude $\lambda$ :

$$
\begin{aligned}
& \left\langle 0\left|\eta_{\Sigma 0}(0)\right| \Sigma\right\rangle=\lambda_{\Sigma} \nu_{\Sigma}(p), \\
& \left\langle 0\left|\eta_{\Lambda}(0)\right| \Lambda\right\rangle=\lambda_{\Lambda} \nu_{\Lambda}(p),
\end{aligned}
$$

where $\nu(p)$ is a Dirac spinor.

The correlation function $\Pi(p)$ may be expressed as

$$
\begin{aligned}
\left\langle 0\left|T \eta_{\Sigma^{0}}(x) \bar{\eta}_{\Lambda}(0)\right| 0\right\rangle & \\
= & -\frac{2}{\sqrt{3}} i \epsilon^{a b c} \epsilon^{a^{\prime} b^{\prime} c^{\prime}}\left\{\gamma_{5} \gamma^{\mu} S_{s}^{a a^{\prime}}(x) \gamma_{\nu} C\left[S_{u}^{b b^{\prime}}(x)\right]^{T}\right. \\
& \times C \gamma_{\mu} S_{d}^{c c^{\prime}}(x) \gamma^{\nu} \gamma_{5} \\
& \left.-\gamma_{5} \gamma^{\mu} S_{s}^{a a^{\prime}}(x) \gamma_{\nu} C\left[S_{d}^{b b^{\prime}}(x)\right]^{T} C \gamma_{\mu} S_{u}^{c c^{\prime}}(x) \gamma^{\nu} \gamma_{5}\right\}
\end{aligned}
$$

where $i S^{a b}(x)$ is the quark propagator [10]:

$$
\begin{aligned}
i S_{q}^{a b}(x) & =\left\langle 0\left|T\left[q^{a}(x) \bar{q}^{b}(0)\right]\right| 0\right\rangle \\
& =\frac{i \delta^{a b}}{2 \pi^{2} x^{4}} \hat{x}+\frac{i}{32 \pi^{2}} \frac{\lambda_{a b}^{n}}{2} g_{s} G_{\mu \nu}^{n} \frac{1}{x^{2}}\left(\sigma^{\mu \nu} \hat{x}+\hat{x} \sigma^{\mu \nu}\right)
\end{aligned}
$$




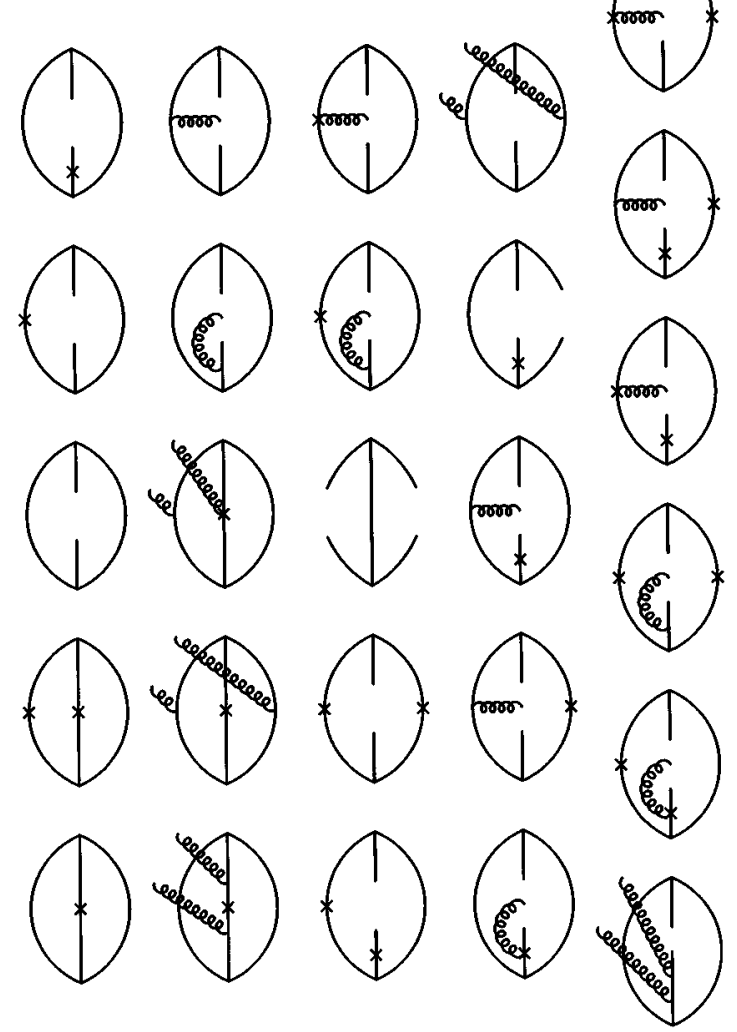

FIG. 1. Relevant diagrams in the QCD sum rules analysis of $\Sigma^{0}$ $\Lambda$ mixing up to dimension seven. The current quark mass correction is denoted by a cross. If two crosses appear in the same diagram, one of them comes from the strange quark.

$$
\begin{aligned}
& -\frac{\delta^{a b}}{12}\langle\bar{q} q\rangle+\frac{\delta^{a b} x^{2}}{192}\left\langle g_{s} \bar{q} \sigma \cdot G q\right\rangle \\
& -\frac{\langle\bar{q} q\rangle\left\langle g_{s}^{2} G^{2}\right\rangle x^{4}}{2^{9} \times 3^{3}} \delta^{a b}-\frac{m_{q} \delta^{a b}}{4 \pi^{2} x^{2}} \\
& +\frac{m_{q}}{32 \pi^{2}} g_{s} \frac{\lambda_{a b}^{n}}{2} G_{\mu \nu}^{n} \sigma_{\mu \nu} \ln \left(-x^{2}\right) \\
& -\frac{\delta^{a b}\left\langle g_{c}^{2} G^{2}\right\rangle}{2^{9} \times 3 \pi^{2}} m_{q^{2}} \ln \left(-x^{2}\right)+\frac{i \delta^{a b} m_{q}\langle\bar{q} q\rangle}{48} \hat{x} \\
& -\frac{i m_{q}\left\langle g_{s} \bar{q} \sigma \cdot G q\right\rangle \delta^{a b} x^{2} \hat{x}}{2^{7} \times 3^{2}}+\cdots
\end{aligned}
$$

At the hadronic level,

$$
\Pi(p)=(-) \lambda_{\Sigma^{0}} \lambda_{\Lambda} \frac{\left(\hat{p}+m_{\Sigma^{0}}\right) \theta\left(p^{2}\right)\left(\hat{p}+m_{\Lambda}\right)}{\left(p^{2}-m_{\Sigma^{0}}^{2}+i \epsilon\right)\left(p^{2}-m_{\Lambda^{0}}^{2}+i \epsilon\right)} .
$$

The diagrams with nonzero contribution are presented in Fig. 1. In the limit of exact isospin symmetry $\Pi(p)$ vanishes. There are two isospin symmetry breaking parameters $\delta m$ and $\gamma$. Each diagram is either propotional to $\delta m$ or to $\gamma$. We explicitly keep the current quark mass term in the quark propagator up to order $O\left(m_{q}\right)$, which is denoted by a cross. The current quark mass enters a diagram either through expanding the free quark propagator up to $O\left(m_{q}\right)$ or through the equation of motion. Since the strange quark mass is not small, we keep terms such as $m_{s}\left(m_{d}-m_{u}\right)$. The calculation is standard as in the QCD sum rule analysis of the baryon mass. Equating the correlator $\Pi(p)$ at the quark level and $\Pi(p)$ at the hadronic level we arrive at two sum rules corresponding to two different structures 1 and $\hat{p}$. Here we present the final result after Borel transformation.

For structure 1,

$$
\begin{aligned}
& \frac{1}{\sqrt{3}}\left\{\delta m M_{B}^{8} E_{3} L^{-8 / 9}+\gamma a M_{B}^{6} E_{2}-\frac{b}{8} \delta m M_{B}^{4} E_{1} L^{-8 / 9}\right. \\
& -\frac{4}{3} \delta m a_{s} m_{s} M_{B}^{4} E_{1} L^{-8 / 9}-\frac{1}{3} \gamma m_{s} a a_{s} M_{B}^{2} E_{0} \\
& +\frac{1}{3} \delta m a^{2} M_{B}^{2} E_{0}-\frac{1}{72} \gamma b a M_{B}^{2} E_{0} \\
& +\frac{7}{48} \delta m a_{s} m_{s} m_{0}^{2} M_{B}^{2} E_{0} L^{-38 / 27} \\
& \left.-\frac{5}{8} \delta \operatorname{mam}_{0}^{2} m_{s} M_{B}^{2} \ln \frac{M_{B}^{2}}{\mu^{2}} E_{0} L^{-38 / 27}\right\} \\
& =(2 \pi)^{4} \lambda_{\Sigma} \lambda_{\Lambda} e^{-m^{2} / M_{B}^{2} 2 m^{2}} \theta\left(m_{\Sigma^{0}}^{2}\right)\left(1+A_{1} M_{B}^{2}\right) .
\end{aligned}
$$

For structure $\hat{p}$,

$$
\begin{aligned}
-\frac{1}{\sqrt{3}}\left\{\delta m m_{s} M_{B}^{6} E_{2} L^{-4 / 3}+4 \gamma m_{s} a M_{B}^{4} E_{1} L^{-4 / 9}\right. \\
\quad+4 \delta m a_{s} M_{B}^{4} E_{1} L^{-4 / 9}+\frac{2}{3} \gamma a a_{s} M_{B}^{2} E_{0} L^{4 / 9} \\
\quad-\frac{1}{4} \gamma m_{s} a m_{0}^{2} M_{B}^{2} E_{0} L^{-26 / 27}-\frac{1}{4} \delta m a_{s} m_{0}^{2} M_{B}^{2} E_{0} L^{-26 / 27} \\
\left.\quad+\frac{1}{8} \delta m a m_{0}^{2} M_{B}^{2} E_{0} L^{-26 / 27}\right\} \\
=(2 \pi)^{4} \lambda_{\Sigma} \lambda_{\Lambda} 2 m e^{-m^{2} / M_{B}^{2}} \theta\left(m_{\Sigma^{0}}^{2}\right)\left(1+A_{2} M_{B}^{2}\right)
\end{aligned}
$$

where $\delta m=m_{d}-m_{u}$ and $\gamma=\langle\bar{d} d\rangle /\langle\bar{u} u\rangle-1, \quad m=m_{\Sigma^{0}}$ $+m_{\Lambda} / 2=1.15 \mathrm{GeV}$ is the average mass. $m_{s}=150 \mathrm{MeV}$ is the strange quark mass. $y=W^{2} / M_{B}^{2}$ and the factors $E_{n}(y)$ $=1-e^{-y} \sum_{k=0}^{n}(1 / k !) y^{k}$, are used to subtract the continuum contribution [11]. $W^{2}=3.4 \mathrm{GeV}^{2}$ is the continuum threshold which is determined together with the overlap amplititude $(2 \pi)^{4} \lambda_{\Sigma}^{2}=1.88 \mathrm{GeV}^{6},(2 \pi)^{4} \lambda_{\Lambda}^{2}=1.64 \mathrm{GeV}^{6}$ from the mass sum rules $[12,13]$. We adopt the "standard" values for the various condensates $b=\left\langle 0\left|g_{s}^{2} G^{2}\right| 0\right\rangle=0.474 \mathrm{GeV}^{4}, \quad a=$ $-(2 \pi)^{2}\langle 0|\bar{u} u| 0\rangle=0.55 \quad \mathrm{GeV}^{3}, \quad a_{s}=-(2 \pi)^{2}\langle 0|\bar{s} s| 0\rangle$ $=0.55 \times 0.8 \quad \mathrm{GeV}^{3}, \quad a m_{0}^{2}=(2 \pi)^{2} g_{s}\langle 0|\bar{u} \sigma \cdot G u| 0\rangle, \quad m_{0}^{2}$ $=0.8 \mathrm{GeV}^{2} . L=\ln \left(M_{B} / \Lambda_{\mathrm{QCD}}\right) / \ln \left(\mu / \Lambda_{\mathrm{QCD}}\right), \Lambda_{\mathrm{QCD}}$ is the 
QCD parameter, $\Lambda_{\mathrm{QCD}}=100 \mathrm{MeV}, \mu=0.5 \mathrm{GeV}$ is the normalization point to which the used values of condensates are referred.

We further improve the numerical analysis by taking into account the renormalization group evolution of the sum rules (11) and (12) through the anomalous dimensions of the various condensates and currents. $A_{1}$ and $A_{2}$ are constants to be determined from the sum rule. They arise from the mixing with the excited states $\Sigma^{0 *}-\Lambda$ or $\Sigma^{0}-\Lambda^{*}$ which were first introduced in the QCD sum rules analysis of the nucleon magnetic moments [11]. The working interval for the Borel mass $M_{B}^{2}$ is $1.3 \mathrm{GeV}^{2} \leqslant M_{B}^{2} \leqslant 2.5 \mathrm{GeV}^{2}$ where both the continuum contribution and power corrections are controllable. Moving the factor $(2 \pi)^{4} \lambda_{\Sigma} \lambda_{\Lambda} e^{-m^{2} / M_{B}^{2}}$ on the righthand side to the left and fitting the new sum rule with a straight line approximation we may extract the mixing parameter $\theta$.

Various theoretical approaches $[2,10,14-18]$ yield consistent results for the quark mass difference $\delta m=3.2 \pm 0.4$ $\mathrm{MeV}$. The difference of the up and down quark condensate has been analyzed with the chiral perturbation theory [14], the QCD sum rules for scalar and pseudoscalar mesons [1719], effective models of QCD incorporating the dynamical breaking of chiral symmetry [20,21], and the QCD sum rules for baryons [10]. The numerical results from the above approaches are $\gamma=-(6-10) \times 10^{-3}[14],-(10 \pm 3) \times 10^{-3}$ [17], $-(7-9) \times 10^{-3}[20,21]$, and $-6.57 \times 10^{-3}[10]$.

In both of the sum rules (11) and (12) the contribution from $\delta m$ and $\gamma$ has opposite sign. The mixing parameters from Eqs. (11) and (12) would contradict each other if $\gamma$ were too large or too small. In Fig. 2 the Borel mass dependence of the mixing parameter and the fitting straight line is shown. Through the intersection with the $Y$ axis, we can obtain the value of $\theta$ directly. With $\gamma=-(7 \pm 1) \times 10^{-3}$ and

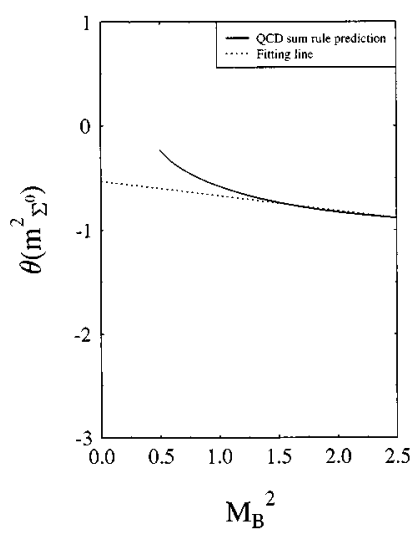

(a)

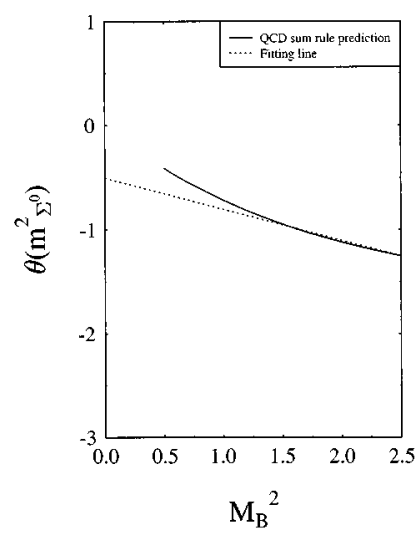

(b)
FIG. 2. (a) The Borel mass dependence of the mixing parameter. The solid curve is the QCD sum rule prediction for $\gamma=$ $-7 \times 10^{-3}$ from Eq. (11]) for structure 1 with $\delta m=3.0 \mathrm{MeV}$ after the numerical factor $(2 \pi)^{4} \lambda_{\Sigma_{0} 0}^{2} \lambda_{\Lambda}^{2} e^{-m^{2} / M_{B}^{2}}$ is moved to the left-hand side. The dotted curve is the fitting straight line. The Borel mass $M_{B}^{2}$ is in unit of $\mathrm{GeV}^{2}$. The mixing parameter $\theta$ is in unit of $\mathrm{MeV}$. (b) The Borel mass dependence of the mixing matrix for the structure $\hat{p}$. The notation is the same as in (a).

$\delta m=3.0 \pm 0.4 \mathrm{MeV}$, our final result is $\theta\left(m_{\Sigma^{0}}^{2}\right)$ $=(-)(0.5 \pm 0.1) \mathrm{MeV}$. It can be seen from Fig. 2 that Eqs. (11) and (12) yield almost the same value for $\theta$. In summary we have calculated the on-shell $\Sigma^{0}-\Lambda$ mixing parameter, which may be measured in future experiments.

This work was supported in part by the Postdoctoral Science Foundation of China and the National Natural Science Foundation of China. It was also supported in part by the National Science Council of R.O.C. (Taiwan) under Grant No. NSC84-2112-M002-021Y.
[1] E. M. Henley and G. A. Miller, in Mesons in Nuclei, edited by M. Rho and D. H. Wilkinson (North-Holland, Amsterdam, 1979), p. 407.

[2] G. A. Miller, B. M. K. Nefkens, and I. Slaus, Phys. Rep. 194, 1 (1990).

[3] L. M. Barkov et al., Nucl. Phys. B256, 365 (1985).

[4] G. A. Miller, in Proceedings of Physics with Light Mesons, edited by W. R. Gibbs and B. M. K. Nefkens, Los Alamos National Laboratory Report No. LA-11184-C, 1987.

[5] D. Alde et al., Z. Phys. C 36, 2189 (1987).

[6] G. Segre and J. Weyers, Phys. Lett. 62B, 91 (1976); M. Oreglia et al., Phys. Rev. Lett. 45, 959 (1980).

[7] D. Coffman et al., Phys. Rev. D 38, 2695 (1988); P. Henrard et al., Nucl. Phys. B292, 670 (1987).

[8] M. A. Shifman, A. I. Vaishtein, and V. I. Zakharov, Nucl. Phys. B147, 385 (1979); B147, 519 (1979).

[9] T. Hatsuda, E. M. Henley, Th. Meissner, and G. Krein, Phys. Rev. C 49, 452 (1994).
[10] K.-C. Yang, W.-Y. Hwang, E. M. Henley, and L. S. Kisslinger, Phys. Rev. D 47, 3001 (1993).

[11] B. L. Ioffe and A. V. Smilga, Nucl. Phys. B232, 109 (1984).

[12] L. J. Reinders, H. Rubinstein, and S. Yazaki, Phys. Lett. 120B, 209 (1983).

[13] W.-Y. Hwang and K.-C. Yang, Phys. Rev. D 49, 460 (1994).

[14] J. Gasser and H. Leutwyler, Phys. Rep. 87, 77 (1982).

[15] S. Weinberg, in A Festschrift for I. I. Rabi (New York Academy of Sciences, New York, 1978).

[16] A. Barducci et al., Phys. Rev. D 38, 238 (1988).

[17] S. Narison, Riv. Nuovo Cimento 10, 1 (1987).

[18] C. A. Dominguez and E. de Rafael, Ann. Phys. (N.Y.) 64, 372 (1987).

[19] S. Narison, Phys. Lett. B 216, 191 (1989).

[20] N. Paver, X. Riazuddin, and M. D. Scadron, Phys. Lett. B 197, 430 (1987).

[21] T. Hatsuda, H. Hogaasen, and M. Prakash, Phys. Rev. C 42, 2212 (1990). 